\begin{tabular}{|l|l|}
\hline Title: & $\begin{array}{l}\text { Transient Trajectory Control of Permanent Magnet Synchronous Machines with } \\
\text { Nonlinear Magnetics }\end{array}$ \\
\hline Authors: & Jan Richter, Philipp Bäuerle, Tobias Gemassmer, Martin Doppelbauer \\
\hline Institute: & $\begin{array}{l}\text { Karlsruhe Institute of Technology (KIT) } \\
\text { Elektrotechnisches Institut (ETI) } \\
\text { Hybrid Electric Vehicles (HEV) }\end{array}$ \\
\hline Type: & Conference Proceedings \\
\hline Published at: & $\begin{array}{l}\text { Proceedings 2015 International Conference on Industrial Technology (ICIT), Seville, } \\
\text { spain, March 17-19, 2015 } \\
\text { Publisher: IEEE, Piscataway (NJ) } \\
\text { Year: } 2015 \\
\text { ISBN: } 978-1-4799-7799-4 \\
\text { Pages: } 2345-2351\end{array}$ \\
\hline Hyperlinks: & DOI: $10.1109 /$ ICIT.2015.7125444 \\
\hline
\end{tabular}

(c) 2015 IEEE. Personal use of this material is permitted. Permission from IEEE must be obtained for all other uses, in any current or future media, including reprinting/republishing this material for advertising or promotional purposes, creating new collective works, for resale or redistribution to servers or lists, or reuse of any copyrighted component of this work in other works. 


\title{
Transient Trajectory Control of Permanent Magnet Synchronous Machines with Nonlinear Magnetics
}

\author{
Jan Richter, Philipp Bäuerle, Tobias Gemassmer, Martin Doppelbauer \\ Karlsruhe Institute of Technology (KIT) \\ Elektrotechnisches Institut (ETI) - Hybrid Electric Vehicles \\ Karlsruhe, Germany \\ Jan.Richter@kit.edu
}

\begin{abstract}
High performance interior permanent magnet synchronous machines show nonlinear magnetics due to saturation and cross-coupling. Nonlinear differential equations describe these phenomena and make feedback control challenging. This paper presents a predictive control method to precisely control the dynamics of these machines. Four real-time capable strategies to online identify transient trajectories are proposed: Two straight line trajectories, a strategy that yields a fast torque response and a strategy that reaches the reference values in a short amount of time. A predictive control method to force the machine to precisely follow the selected trajectory is developed and analyzed using simulations and test bench measurements. Thus, advantages and disadvantages of specific trajectories are identified. This allows the selection of a proper strategy depending on the drive requirements.
\end{abstract}

Keywords-control design; cross-coupling; current control; machine control; nonlinear control systems; permanent magnet machines; predictive control; saturation magnetization; trajectory

\section{INTRODUCTION}

As long as interior permanent magnet synchronous machines (IPMSM) are known, appropriate control algorithms have been developed. Rotor-oriented approaches seem advantageous, because the effects of magnetic anisotropy can easily be described. Moreover, currents, flux linkages and voltages are constant during stationary operation. Analyzing the machine system equations, it becomes apparent that the state of the machine is given by either a current $\left(i_{\mathrm{d}}, i_{\mathrm{q}}\right)$ or a flux linkage $\left(\Psi_{\mathrm{d}}, \Psi_{\mathrm{q}}\right)$ couple. The machine state is directly related to the machine torque. Any control method applies an external voltage $\left(v_{\mathrm{d}}, v_{\mathrm{q}}\right)$ to force the machine state and therefore the currents, flux linkages and the torque to the given reference values. The machine behavior can thus be visualized in the current, flux linkage or voltage plane as given in Fig. 1.

Based on that knowledge numerous control methods have been developed. Depending on the underlying algorithm the currents, flux linkages or the torque are directly controlled involving specific advantages and disadvantages. Whatever method is used, two cases can always be distinguished: First the case that the controller voltage demand can be realized by the inverter and second the case that the controller voltage demand is higher than the maximal inverter output voltage. In the first case a dead-beat step, a finite settling time or a PI-type trajectory to the reference values are possible. This is well known and extensively described in literature. The second case
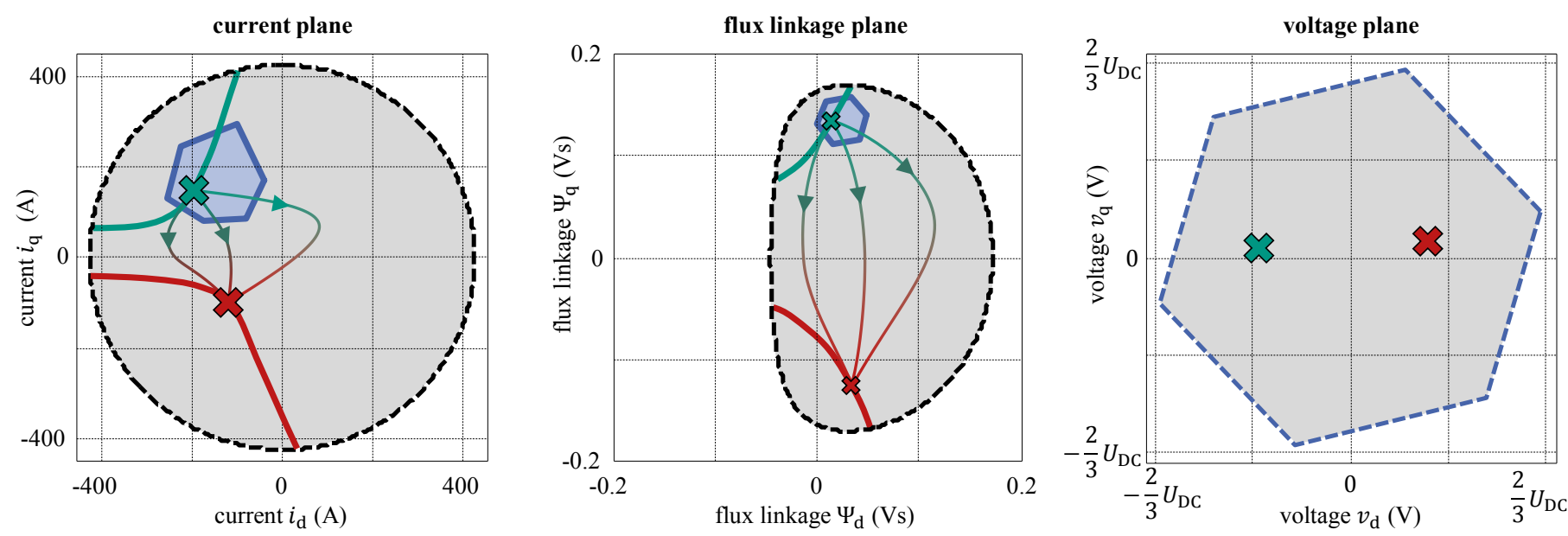

Fig. 1 Current, flux linkage and voltage planes for the machine under test. The area of safe operation is shadowed grey in all planes. The green crosses represent a stationary operation point with a torque of $130 \mathrm{Nm}$ at a speed of $2100 \mathrm{~min}^{-1}$ and a DC link voltage $U_{\mathrm{DC}}$ of $300 \mathrm{~V}$. By application of the inverter output voltage limit for one control period of $125 \mu \mathrm{s}$, operation points on the blue line in the current and flux linkage plane can be reached. Another stationary operation point of new reference values is given by the red crosses. Three transient trajectories connecting the old and new reference values are exemplarily given. Constant torque lines are drawn as green and red lines. 
often occurs when the machine state changes rapidly, especially when the rotor speed is high. Then the demanded voltage has to be truncated. Different methods have been proposed for that purpose.

In the $1990 \mathrm{~s}$ microprocessors with digital controllers enabled truncation schemes in the voltage plane. The demanded voltage is shortened to the inverter hexagon voltage limit with constant phase angle, with constant d-axis voltage or with respect to the back electromotive force [1, 2]. A comparison of the methods for IPMSM can be found in [3]. However, the influence of voltage truncation on currents and flux linkages remains unknown and is not controlled during transients. To overcome that problem the current reference values during dynamic voltage limitation can be adjusted [4]. This simple principle only needs negligible calculation time and yields good results. Though, since the controller cannot follow the adjusted reference values, precise control of the current and flux linkage trajectory is not possible. Thus, methods have been developed that calculate the transient trajectory in an optimal way in respect to a quality function. Since the currents and flux linkages have to be optimized over time a multi-dimensional optimization problem has to be solved. Time optimal control is applied to reach the current [5] and torque [6] reference values quickly. Although settling time is reduced, it often is not of interest to take the fastest trajectory but the one that maximizes the quality function over time. For instance the torque-time area during transients can be more important than the actual time to the torque reference value. This is considered by single step optimization methods as proposed in [7] and [8]. The methods are real-time capable but since they only optimize single steps they might not identify the overall optimal trajectory in respect to the quality function. This is avoided by offline calculation of the optimal trajectory by dynamic programming [9]. Although the best possible trajectory in respect to the quality function is identified, this approach is not real-time capable with today's microprocessors.

All proposed approaches only work for linear machines neglecting the effects of saturation and cross-coupling. In this paper they are extended to machines with nonlinear magnetics. Distortion of the calculated trajectories due to the nonlinear flux linkage relations is considered and schemes to ensure that all machine quantities stay within the safe area of operation are designed. Four strategies to identify proper trajectories are proposed. Two strategies use straight lines to connect the old and new reference values. Another strategy aims at a fast torque response and the last minimizes the amount of time that is needed to reach the reference values. A real-time capable predictive control approach ensures that the machine precisely follows the calculated trajectory. The performance of all four methods is analyzed and validated using simulations and test bench measurements. It is thus shown how transient trajectories can be identified and controlled for machines with nonlinear magnetics in real-time.

\section{THEORY}

Starting with the machine system equations the graphs in Fig. 1 are developed step by step and the consequences of nonlinear magnetics to the machine behavior are introduced.
The machine is assumed to have three symmetric starconnected phases with the neutral point not connected to the inverter. Dielectric currents, temperature dependencies as well as skin and proximity effects are neglected. Moreover, iron and friction losses are disregarded. The stator voltages can be calculated by employing Ohm's law, Faraday's law of induction and Kirchhoff's laws to the machine's coils. Subsequent transformation to the rotor-fixed dq-frame yields:

$$
\begin{aligned}
& v_{\mathrm{d}}=R i_{\mathrm{d}}+\frac{\mathrm{d} \Psi_{\mathrm{d}}}{\mathrm{d} t}-\omega \Psi_{\mathrm{q}} \\
& v_{\mathrm{q}}=R i_{\mathrm{q}}+\frac{\mathrm{d} \Psi_{\mathrm{q}}}{\mathrm{d} t}+\omega \Psi_{\mathrm{d}}
\end{aligned}
$$

There $R$ denotes the ohmic stator resistance, $\omega$ the electric frequency, $t$ the time and $v_{k}, i_{k}$ and $\Psi_{k}$ the voltages, currents and flux linkages in the direct and quadrature axes with $k \in$ $\{d, q\}$. The flux linkages depend nonlinearly on the currents to account for saturation and cross-coupling. The relations are given by the functions $f$ and $g$ and shown in Fig. 7 for the machine under test. Both functions are invertible which follows the given assumptions [10].

$$
\begin{aligned}
& \Psi_{\mathrm{d}}=f\left(i_{\mathrm{d}}, i_{\mathrm{q}}\right) \text { and } i_{\mathrm{d}}=f^{-1}\left(\Psi_{\mathrm{d}}, \Psi_{\mathrm{q}}\right) \\
& \Psi_{\mathrm{q}}=g\left(i_{\mathrm{d}}, i_{\mathrm{q}}\right) \text { and } i_{\mathrm{q}}=g^{-1}\left(\Psi_{\mathrm{d}}, \Psi_{\mathrm{q}}\right)
\end{aligned}
$$

Equations (1) and (2) contain three electro-magnetic quantities: currents, flux linkages and voltages. That is why the stationary and dynamic behavior of the machine can be analyzed by the three planes in Fig. 1. There, grey colored areas bounded by a dashed line indicate the safe area of operation. The maximal inverter output voltage limits the machine voltage which is given by a rotating hexagon in the dq-frame. The thermal and demagnetization limits of the machine give the maximal current which is a circle in the current plane. Using (3) and (4) the current limit can be converted to a flux linkage limit. In the flux linkage plane the safe area of operation is a distorted ellipsoid due to the nonlinear flux linkage relations. The physical state of the machine is given by a point in the current or flux linkage plane as depicted by the green crosses. The machine state can be defined by either currents or flux linkages because both quantities are uniquely mapped by (3) and (4). It should be kept in mind that the crosses in Fig. 1 show the instantaneous values of the space vectors at one distinct point of time.

The state of the machine is manipulated by the inverter output voltage. When the state given by the green crosses should stay constant, the green space vector in the voltage plane in Fig. 1 has to be applied. It can be calculated by (1) and (2) with the time derivatives set to zero. If the state of the machine is supposed to change, any other voltage space vector is applied. With application of the maximal inverter output voltage the state is changed at a maximal rate. That corresponds to a voltage space vector on the blue dashed hexagon in the voltage plane. Using the voltage space vectors and the actual state of the machine, the reachable flux linkages within one control period can be predicted by (5) and (6).

$$
\begin{aligned}
\Psi_{\mathrm{d}, t_{1}}= & \Psi_{\mathrm{d}, t_{0}}+T_{\mathrm{P}} \cdot \frac{v_{\mathrm{d}, t_{0}}-R \cdot i_{\mathrm{d}, t_{0}}+\omega \Psi_{\mathrm{q}, t_{0}}}{1+\frac{1}{4} \omega^{2} T_{\mathrm{P}}^{2}} \\
& +T_{\mathrm{P}}^{2} \cdot \frac{\frac{1}{2} \omega v_{\mathrm{q}, t_{0}}-\frac{1}{2} \omega R i_{\mathrm{q}, t_{0}}-\frac{1}{4} \omega^{2} \Psi_{\mathrm{d}, t_{0}}}{1+\frac{1}{4} \omega^{2} T_{\mathrm{P}}^{2}}
\end{aligned}
$$




$$
\begin{aligned}
\Psi_{\mathrm{q}, t_{1}} & =\Psi_{\mathrm{q}, t_{0}}+T_{\mathrm{P}} \cdot \frac{v_{\mathrm{q}, t_{0}}-R \cdot i_{\mathrm{q}, t_{0}}-\omega \Psi_{\mathrm{d}, t_{0}}}{1+\frac{1}{4} \omega^{2} T_{\mathrm{P}}^{2}} \\
& +T_{\mathrm{P}}^{2} \cdot \frac{-\frac{1}{2} \omega v_{\mathrm{d}, t_{0}}+\frac{1}{2} \omega R i_{\mathrm{d}, t_{0}}-\frac{1}{4} \omega^{2} \Psi_{\mathrm{q}, t_{0}}}{1+\frac{1}{4} \omega^{2} T_{\mathrm{P}}^{2}}
\end{aligned}
$$

The equations are a time-discrete solution of (1) and (2) with $T_{\mathrm{P}}$ as control period duration and the approximations that the dynamic ohmic voltage drops are negligible and that the flux linkages change linearly. The validity of both approximations for the machine under test is given in [10]. Equations (5) and (6) consider dynamic changes of the back electromotive force although this effect is often neglected $[4,5,7,8,9]$. It is caused by changes of the flux linkages within the control period. If this effect is neglected for the machine under test model calculation errors are severe at higher speeds which is why the precise calculation is used in the following. In (5) and (6) values with an index $t_{0}$ indicate values at the beginning of the control period as given by the green crosses in the current and flux linkage plane in Fig. 1. Values with an index $t_{1}$ are predicted values at the end of the control period. As shown in Fig. 1, application of voltage space vectors on the hexagon border for one control period yields predicted flux linkages on the blue line in the flux linkage plane. There, the green cross is not in the center of the hexagon due to the influence of the back electromotive force. Moreover, the hexagon is slightly distorted due to the change of the back electromotive force and cross-coupling. Subsequent application of (3) and (4) gives the corresponding predicted currents as shown by the blue line in the current plane. Due to saturation and cross-coupling the hexagon is strongly distorted and unsymmetrical. In summary, the voltage hexagon limit can be transformed to a limit of reachable flux linkages and currents by (3) to (6).

The red crosses in Fig. 1 indicate new reference values. They are assumed to be known, either by an offline calculated lookup table or by an online optimal operation method. The new reference values cannot be reached within one control period because they lie outside the blue shapes in the current and flux linkage plane. The change in reference values corresponds to a torque change as indicated by the hyperbolas of constant torque. The torque line of the actual operation point

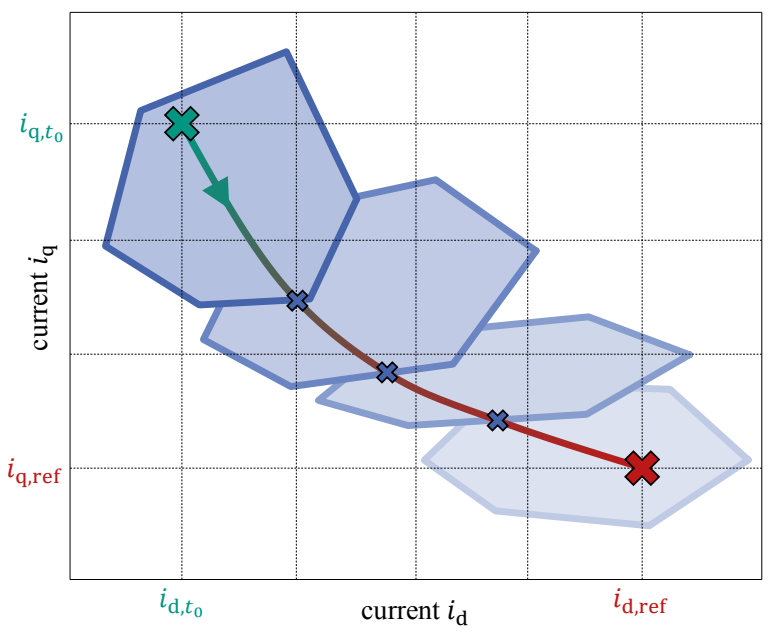

Fig. 2 The transient trajectory is put together of calculations of several control periods. The blue shapes change with the DC link voltage, the rotor angle change and the operation point. is drawn in green and the one of the new reference point in red. The new reference values can be reached by different transient trajectories given as arbitrary nonlinear lines connecting the green and red crosses in the current or flux linkage plane in Fig. 1. As shown in Fig. 2 calculations of several control periods are merged to form the transient trajectory over time. In the example of Fig. 2 the maximal inverter output voltage is used three times before a dead-beat step is performed. Thus, a point on the border of the blue shape has to be selected for the first three time steps. Depending on what expectations one has to the drive performance different points can be chosen yielding different trajectories. In the following four strategies are proposed that identify optimal points $\left(i_{\mathrm{d}}^{*}, i_{\mathrm{q}}^{*}\right)$ in each time step in respect to a quality function. Two methods use straight line trajectories, one gives a fast torque response and the last minimizes the time to the reference values. Since all strategies optimize the controller output voltage of a single time step, they can be classified as single step optimization methods. Such an approach does not necessarily identify the best overall trajectory for the given quality function. However, implementation is easy and real-time capable with nonlinear magnetics of the machine being taken into account.

\section{A. Direct Current Connection (DCC)}

It is reasonable to ensure that both the d-and q-current stay in between the old and new reference values at all times. Thereby over-currents are avoided even during transients. A trajectory reaching that goal is a direct straight line from the old to the new reference value as depicted in Fig. 3 (a). The actual current is marked by a green cross and the new reference value by a red cross. In order to calculate the respective controller output voltage, the flux linkages and currents are predicted by (3) to (6) for the six corners $l \in\{1,2,3,4,5,6\}$ of the voltage hexagon. The predicted currents are given by the small blue crosses in Fig. 3 (a). Straight lines connect the six points. This approximation is sufficiently accurate for the machine under test. It should be noted that more points could be calculated for higher accuracy with higher calculation effort. By simple calculations of analytic geometry the edge that intersects the wished straight trajectory is found and the
A. Direct Current Connection (DCC)

(a)

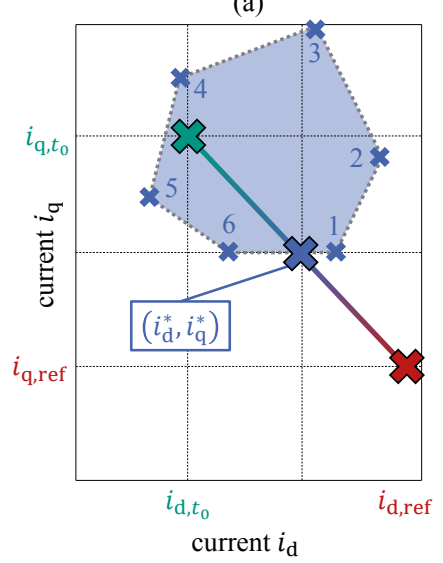

$B$. Direct Flux Linkage Connection (DFLC) (b)

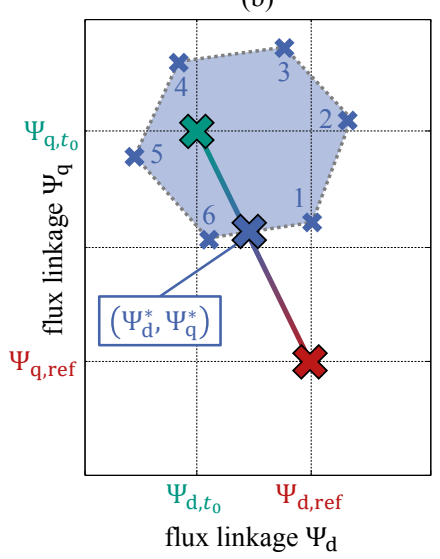

Fig. 3 Scheme of the direct current connection (a) and the direct flux linkage connection (b). The currents $\left(i_{\mathrm{d}}^{*}, i_{\mathrm{q}}^{*}\right)$ are selected by intersection of one edge and the direct straight line from the actual to the reference value. 
currents $i_{\mathrm{d}}^{*}$ and $i_{\mathrm{q}}^{*}$ of the intersection point can be calculated. The controller output voltage then is:

$$
\begin{aligned}
& v_{\mathrm{d}}=R i_{\mathrm{d}, t_{0}}+\frac{f\left(i_{\mathrm{d}}^{*}, i_{\mathrm{q}}^{*}\right)-\Psi_{\mathrm{d}, t_{0}}}{T_{\mathrm{P}}}-\frac{1}{2} \omega\left(\Psi_{\mathrm{d}, t_{0}}+f\left(i_{\mathrm{d}}^{*}, i_{\mathrm{q}}^{*}\right)\right) \\
& v_{\mathrm{q}}=R i_{\mathrm{q}, t_{0}}+\frac{g\left(i_{\mathrm{d}}^{*}, i_{\mathrm{q}}^{*}\right)-\Psi_{\mathrm{q}, t_{0}}}{T_{\mathrm{P}}}+\frac{1}{2} \omega\left(\Psi_{\mathrm{q}, t_{0}}+g\left(i_{\mathrm{d}}^{*}, i_{\mathrm{q}}^{*}\right)\right)
\end{aligned}
$$

Equations (7) and (8) follow from (1) and (2) with the approximations introduced in (5) and (6).

\section{B. Direct Flux Linkage Connection (DFLC)}

The same strategy can be used in the flux linkage plane. By controlling the flux linkage trajectory to a straight line the back electromotive force of the machine is controlled. The procedure is very similar to the one described in Section II $A$. Equations (5) and (6) are used to predict the flux linkages for the six voltage hexagon corners. As shown in Fig. 3 (b) the flux linkages $\Psi_{\mathrm{d}}^{*}$ and $\Psi_{\mathrm{q}}^{*}$ that intersect one edge can be derived by means of analytic geometry. The controller output voltage then follows with:

$$
\begin{aligned}
& v_{\mathrm{d}}=R i_{\mathrm{d}, t_{0}}+\frac{\Psi_{\mathrm{d}}^{*}-\Psi_{\mathrm{d}, t_{0}}}{T_{\mathrm{P}}}-\frac{1}{2} \omega\left(\Psi_{\mathrm{d}, t_{0}}+\Psi_{\mathrm{d}}^{*}\right) \\
& v_{\mathrm{q}}=R i_{\mathrm{q}, t_{0}}+\frac{\Psi_{\mathrm{q}}^{*}-\Psi_{\mathrm{q}, t_{0}}}{T_{\mathrm{P}}}+\frac{1}{2} \omega\left(\Psi_{\mathrm{q}, t_{0}}+\Psi_{\mathrm{q}}^{*}\right)
\end{aligned}
$$

It should be noted that both DCC and DFLC automatically avoid over-currents during transients because any straight line connecting valid reference values stays within the safe area of operation in the current or flux linkage plane as can be seen in Fig. 1.

\section{Fast Torque Response (FTR)}

When the focus is set on mechanic performance a trajectory can be chosen in a way that the reference torque is approached as fast as possible. Thereby the torque control deviation is minimized in each time step. This has already been proposed by several authors [7, 8]. In the following the method is extended to machines with nonlinear magnetics including a proper scheme to keep the currents within the safe area of operation.

Just as in Section II $A$ the six voltage hexagon corners are

C. Fast Torque Response (FTR) (a)

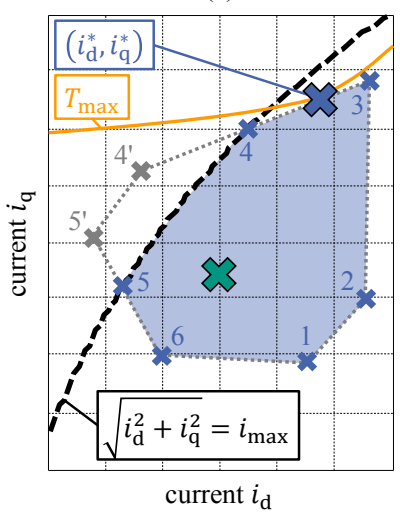

(b)

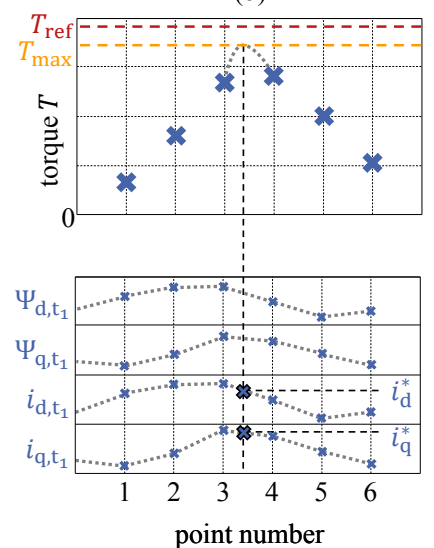

Fig. 4 Scheme for a fast torque response. The over-current protection (a) and the calculation of the optimal torque at each time step (b) are displayed. used to predict the flux linkages $\left(\Psi_{\mathrm{d}, t_{1}}, \Psi_{\mathrm{q}, t_{1}}\right)$ and currents $\left(i_{\mathrm{d}, t_{1}}, i_{\mathrm{q}, t_{1}}\right)$ by (3) to (6). The so predicted currents are shown in Fig. 4 (a). There, two corners lie outside the safe area of operation as drawn by the grey crosses. In order to prevent the trajectory to leave the maximal current circle during transients the two points are scaled back to the two blue crosses on the current limit. The torque $T_{l}$ for the six corner points is calculated with $p$ as the number of pole pairs and $l \in$ $\{1,2,3,4,5,6\}$ as the point number.

$$
T_{l}=\frac{3}{2} p \cdot\left(\Psi_{\mathrm{d} l, t_{1}} i_{\mathrm{q} l, t_{1}}-\Psi_{\mathrm{q} l, t_{1}} i_{\mathrm{d} l, t_{1}}\right)
$$

The so calculated torque is depicted in Fig. 4 (b) in the upper plot. The point that approaches the reference torque as close as possible has to be identified next. Simple selection of the maximum point is not accurate enough because the maximum does not necessarily lie on one of the points. Consequently, the torque in between the points has to be computed after the relevant section is identified. In the example of Fig. 4 (b) the relevant section is the one between points 3 and 4 . In the lower plot of Fig. 4 (b) the predicted flux linkages and currents are drawn as blue crosses for all six points. The values in between the crosses can be linear interpolated as given by the straight grey dashed lines. When the straight line equations are inserted into (11) a polynomial function of degree two is obtained. By means of differential calculus the respective maximum can be found. An analytic expression only dependent on the four predicted currents and flux linkages of the adjacent points can be derived. This procedure yields the currents $i_{\mathrm{d}}^{*}$ and $i_{\mathrm{q}}^{*}$ that are marked by the big blue cross in Fig. 4 (a). It can be seen that this is the only current point that lies on the hyperbola of the maximal reachable torque. Using (7) and (8) the respective controller output voltage is calculated.

\section{Short Time to Reference Values (STRV)}

It might be of interest to use a trajectory that reaches the reference values in a short amount of time. In order to identify such a trajectory the voltage $v_{\mathrm{DB}}$ that reaches the reference values with a dead-beat step can be used as a quality function. Although the dead-beat step cannot be performed, this voltage is a measure for the future voltage demand to reach the reference values. If the future voltage demand is small, then the

D. Short Time to Reference Values (STRV) (a)

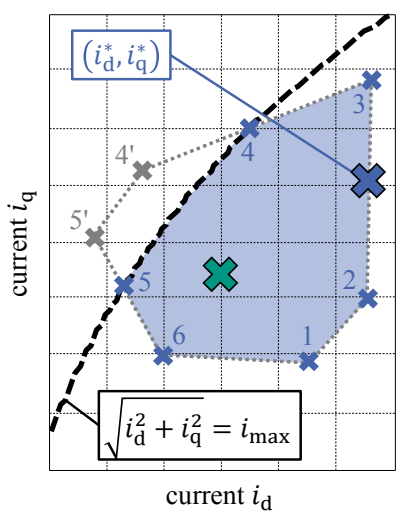

(b)

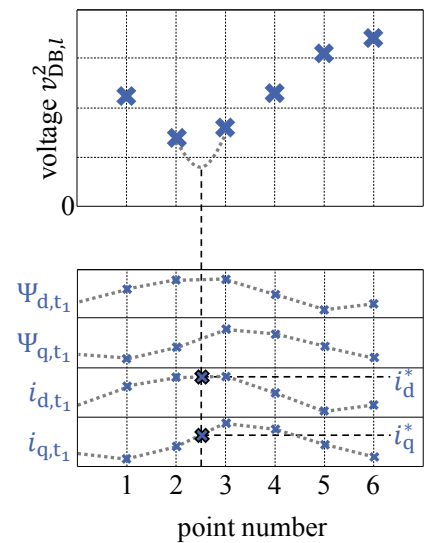

Fig. 5 Scheme for a short time to the reference values. The over-current protection (a) and the voltage calculation at each time step (b) are displayed. 
needed overall time to the reference values will also be. Hence, the reachable current point with the smallest dead-beat step voltage is chosen in each time step which is calculated in the following.

The procedure is very similar to the one described in Section II $C$. The six voltage hexagon corners are transformed to the flux linkage and current plane by (3) to (6) as shown in Fig. 5 (a). The current limitation scheme is identical to the one used in FTR. However, a different quality function is used. With the approximations introduced in (5) and (6) the square of the dead-beat step voltage amplitude $v_{\mathrm{DB}, l}^{2}$ can be calculated for each point by:

$$
\begin{gathered}
v_{\mathrm{d} l}=R i_{\mathrm{d} l, t_{0}}+\frac{\Psi_{\mathrm{d}, \mathrm{ref}}-\Psi_{\mathrm{d} l, t_{1}}}{T_{\mathrm{P}}}-\frac{1}{2} \omega\left(\Psi_{\mathrm{d} l, t_{1}}+\Psi_{\mathrm{d}, \mathrm{ref}}\right) \\
v_{\mathrm{q} l}=R i_{\mathrm{q} l, t_{0}}+\frac{\Psi_{\mathrm{q}, \mathrm{ref}}-\Psi_{\mathrm{q} l, t_{1}}}{T_{\mathrm{P}}}+\frac{1}{2} \omega\left(\Psi_{\mathrm{q} l, t_{1}}+\Psi_{\mathrm{q}, \mathrm{ref}}\right) \\
v_{\mathrm{DB}, l}^{2}=v_{\mathrm{d} l}^{2}+v_{\mathrm{q} l}^{2}
\end{gathered}
$$

There $\Psi_{\mathrm{d} \text {,ref }}$ and $\Psi_{\mathrm{q}, \mathrm{ref}}$ denote the reference flux linkages. The so determined value $v_{\mathrm{DB}, l}^{2}$ is given for the six points in the upper plot of Fig. 5 (b). The minimum of $v_{\mathrm{DB}, l}^{2}$ has to be calculated next. This is done using the same approach and approximations as in Section II $C$. The values of the predicted currents and flux linkages of the six points are linear connected as shown by the dashed grey lines in the lower plot of Fig. 5 (b). If the straight line equations of the relevant section are inserted into (12) to (14) a polynomial function of degree two is obtained. Minimization using differential calculus yields an analytic expression that is only dependent on the four currents and flux linkages of the adjacent points. In this way the currents $i_{\mathrm{d}}^{*}$ and $i_{\mathrm{q}}^{*}$ are determined. They are marked by the big blue cross in Fig. 5 (a). By (7) and (8) the corresponding controller output voltage is calculated.

\section{EXPERIMENTAL SETUP}

A motor test bench as shown in Fig. 6 is used to evaluate the algorithms described in Section II. Measurements are conducted with an interior permanent-magnet synchronous machine of type Brusa HSM1-6.1712-CO1. The properties of

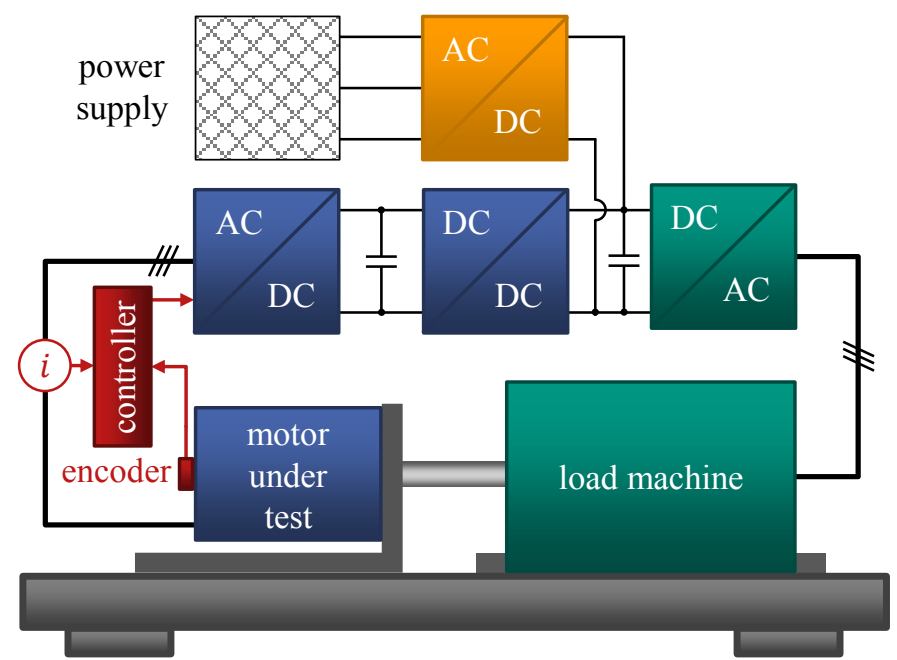

Fig. 6 Test bench setup used for the experimental evaluation of the described methods.
TABLE I. MACHINE PROPERTIES

\begin{tabular}{|l|c|}
\hline Voltage nom. & $212 \mathrm{~V}$ \\
\hline Current nom. / max. & $169 \mathrm{~A} / 300 \mathrm{~A}$ \\
\hline Shaft power nom. / max. & $57 \mathrm{~kW} / 97 \mathrm{~kW}$ \\
\hline Speed nom. / max. & $4200 \mathrm{~min}^{-1} / 11000 \mathrm{~min}^{-1}$ \\
\hline Torque nom. / max. & $130 \mathrm{Nm} / 220 \mathrm{Nm}$ \\
\hline Ohmic stator resistance typ. & $10.5 \mathrm{~m} \Omega$ \\
\hline Number of pole pairs & 3 \\
\hline
\end{tabular}

the machine under test are given in Table I. An asynchronous machine by Wittur is used as load. Inverters for grid connection and control of both machines are based on Semikron SkiiP 513GD122-3DUL modules. Currents are measured by the built-in Semikron SkiiP current transducers. Speed and rotor angle are calculated using the machine's built-in incremental encoder signals. The control methods described in Section II are implemented on the digital signal processor (DSP) TMS320C6748 produced by Texas Instruments. The flux linkage functions $f$ and $g$ depicted in Fig. 7 are stored as lookup tables in an external SD-RAM. To realize a switching frequency of $8 \mathrm{kHz}$ all control algorithm calculations are executed within less than $125 \mu$ s. Inverter gate signals are created by space vector modulation using a field programmable gate array (FPGA) of the Cyclone series by Altera.

\section{RESULTS AND DISCUSSION}

After the identification of the flux linkage functions is described, the performance of the four algorithms given in Section II is analyzed for a torque step and a torque inversion.

\section{A. Identification of Flux Linkage Functions}

The flux linkage functions have to be known for the machine under test so that the control algorithm calculations can be executed. The flux linkages can be identified by stationary measurements at constant speed [11]. Then (1) and (2) can be solved for the flux linkages with all other quantities being measured. This can be repeated in the whole dq-current plane resulting in the graphs of Fig. 7. Flux linkage observers can be used to cope with parameter variations during real
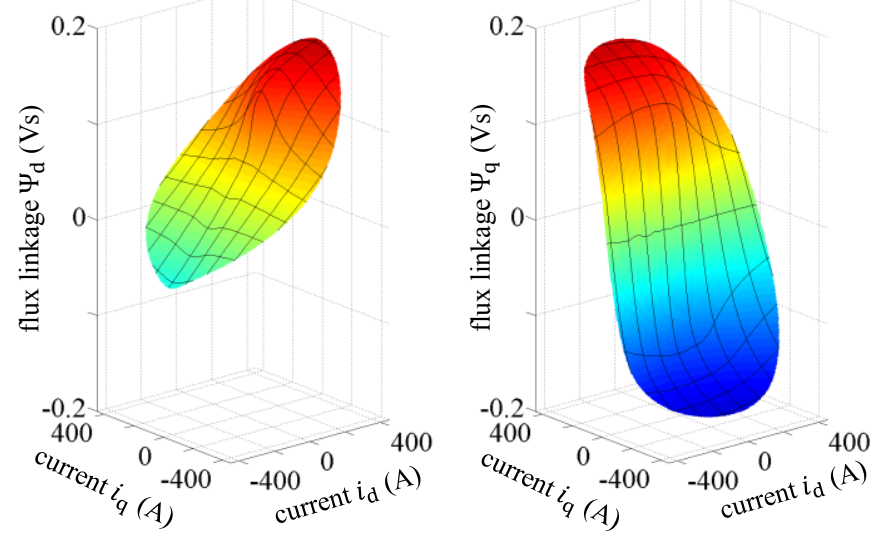

Fig. 7 Measured flux linkage functions $\Psi_{\mathrm{d}}=f\left(i_{\mathrm{d}}, i_{\mathrm{q}}\right)$ and $\Psi_{\mathrm{q}}=g\left(i_{\mathrm{d}}, i_{\mathrm{q}}\right)$ for the machine under test in the whole operational area. Nonlinearities due to saturation and cross-coupling are clearly visible. 
operation [12].

\section{B. Torque Step}

The described methods are analyzed by means of a torque step from no load to $200 \mathrm{Nm}$ at half of the nominal speed as shown in Fig. 8. Measurement results are given on the top and according simulations on the bottom. Transient trajectories of the currents and flux linkages for all methods can be seen. The torque developing calculated by (11) is given as well. Agreement of measurements and simulations is astonishing proving the validity of the used equations and approximations. Moreover, it should be noted that the machine dynamics are precisely simulated by using flux linkage functions that are identified by stationary measurements. However, the measured torque rises slower in comparison to the simulation. This can be explained by dynamic changes of the DC link voltage $U_{\mathrm{DC}}$. It is assumed to be constant in the simulation whereas it significantly drops in the real measurement due to the large dynamic power demand. That is why a smaller voltage is applied in the real measurement yielding a slower torque rise.

As expected DCC results in a straight line trajectory in the current plane and DFLC in the flux linkage plane. STRV and FTR decrease the d-current before the q-current is increased. FTR decreases the d-current until the reference torque hyperbola is reached. Though, the FTR trajectory hits the maximal current limit and is bounded until the torque hyperbola is met. Thereby over-currents are avoided even during transients and in the presence of saturation and crosscoupling. The current reference values are reached within 17 control periods by DFLC and STRV as given in Table II. Although FTR is slower in respect to the time to reach the current reference values, the produced torque along the
TABLE II.

METHOD COMPARISON

\begin{tabular}{|c|c|c|c|c|}
\hline \multirow{2}{*}{ Method } & \multicolumn{2}{|c|}{ Torque Step } & \multicolumn{2}{c|}{ Torque Inversion } \\
\cline { 2 - 5 } & t to $\boldsymbol{i}$ ref. & M-t area & $\boldsymbol{t}$ to $\boldsymbol{i}$ ref. & M-t area \\
\hline \multirow{2}{*}{ DCC } & $22 T_{\mathrm{P}}$ & $40.0 \%$ & $19 T_{\mathrm{P}}$ & $69.0 \%$ \\
\hline DFLC & $17 T_{\mathrm{P}}$ & $48.5 \%$ & $20 T_{\mathrm{P}}$ & $67.2 \%$ \\
\hline FTR & $18 T_{\mathrm{P}}$ & $55.4 \%$ & $32 T_{\mathrm{P}}$ & $49.2 \%$ \\
\cline { 3 - 5 } STRV & $17 T_{\mathrm{P}}$ & $52.5 \%$ & $17 T_{\mathrm{P}}$ & $70.3 \%$ \\
\hline
\end{tabular}

trajectory is bigger as can be seen in the torque developing in Fig. 8. In order to precisely compare the torque performance of the four methods the torque-time area during transient operation is calculated. The results are normalized to the area of the grey rectangle in the measured torque plot in Fig. 8. As given in Table II the torque-time area of FTR has the biggest value and thus yields the best torque response in this experiment.

\section{Torque Inversion}

The performance of the methods significantly changes when a torque inversion is analyzed. Measurement and simulation results for a torque inversion from $-200 \mathrm{Nm}$ to $200 \mathrm{Nm}$ are given in Fig. 9. Again DCC connects the old and new reference values with a straight line. In this example the torque inversion corresponds to an inversion of the q-current with the d-current staying constant. Although the machine is operated in the nonlinear regime and the q-current is changed at its maximal rate the d-current is almost kept constant. Thus, the currents in both axes can be controlled independently even during transients. By DCC the machine's d- and q-axis are thus dynamically decoupled. STRV again decreases the d-current with the trajectory being bounded to the maximal current limit.
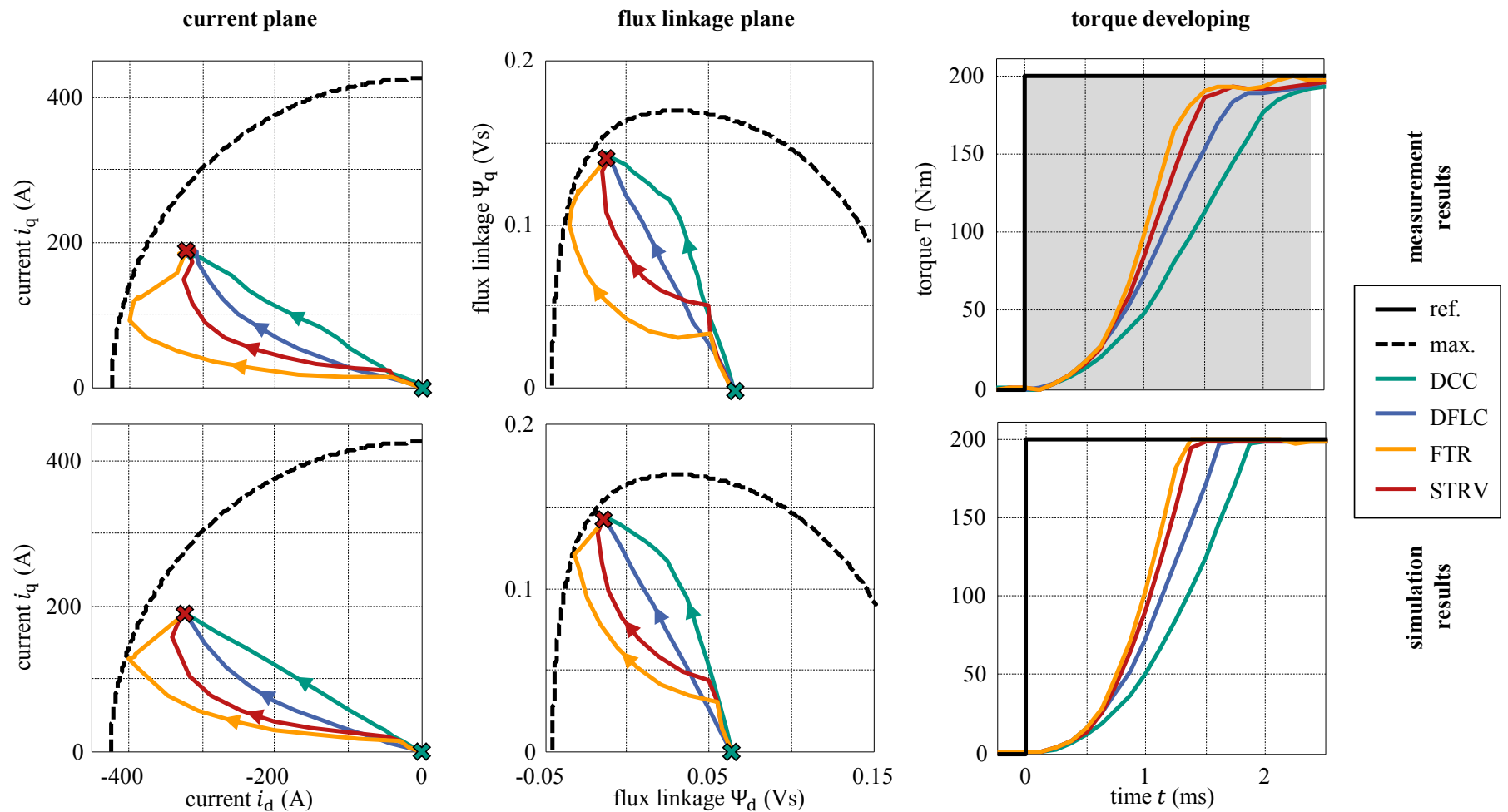

Fig. 8 Torque step from $0 \mathrm{Nm}$ to $200 \mathrm{Nm}$ at $2100 \mathrm{~min}^{-1}$ with a DC link voltage $U_{\mathrm{DC}}$ of $300 \mathrm{~V}$. The trajectories in the current and flux linkage plane are shown as well as the torque developing for all methods. Measurements are given on the top and according simulation results on the bottom. 

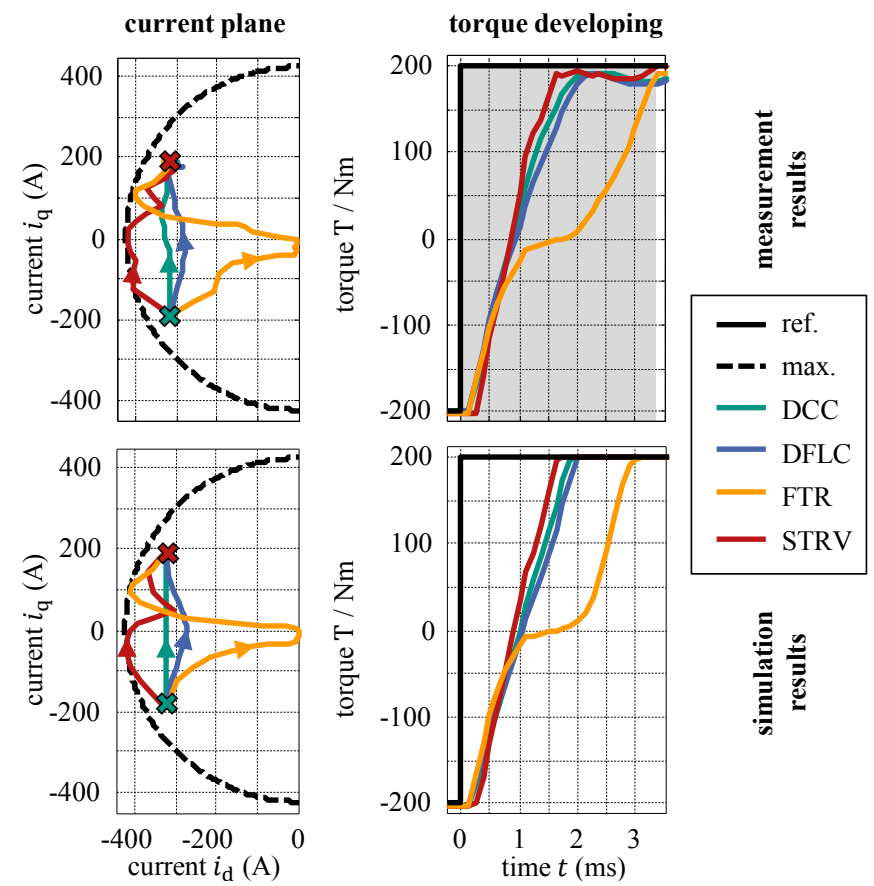

Fig. 9 Torque inversion from $-200 \mathrm{Nm}$ to $200 \mathrm{Nm}$ at $2100 \mathrm{~min}^{-1}$ with a DC link voltage $U_{\mathrm{DC}}$ of $300 \mathrm{~V}$. The trajectories in the current plane and the torque developing are shown for all methods. Measurements are given on the top and according simulation results on the bottom.

This reduces the back electromotive force and allows the reference values to be reached in a shorter amount of time as given in Table II. FTR is the slowest method for the torque inversion. This is due to the fact that the d-current is increased in the first control periods. Although this increases the torque at the fastest rate at first, the increasing back electromotive force severely slows down the torque increase afterwards. This can be seen in the torque developing in Fig. 9 and in the calculated torque-time area in Table II. Consequently FTR is not suitable for reference torque steps with a sign change. A possible solution is to switch to any other method if a torque sign change is detected.

\section{CONCLUSION}

A predictive algorithm for precise control of the dynamics of interior permanent magnet synchronous machines with nonlinear magnetics is covered in this contribution. Iron saturation, cross-coupling and the magnetic anisotropy of the machine are taken into account. Four different strategies to identify transient trajectories are proposed. Two strategies use straight line trajectories, another gives a fast torque response and the last minimizes the time to reach the reference values. All methods limit the controller output voltage to the hexagon border. The straight line strategies are easy to implement and require little calculation time. However, measurements prove that they are not optimal in respect to mechanic performance. An improved torque response is realized using a trajectory that minimizes the torque control deviation in each time step.
However, if the sign of the reference torque changes a slow electric and mechanic response is obtained. This is avoided by a strategy that minimizes the time to reach the reference values. In respect to overall performance this method thus proves to be the most suitable which is validated using test bench measurements. In summary the proposed methods enable accurate, real-time capable identification and control of transient trajectories for machines with nonlinear magnetics even in the presence of strong iron saturation and crosscoupling while the drive system is operated at the physical limit of the machine and the inverter.

\section{REFERENCES}

[1] S. Morimoto, M. Sanada, and Y. Takeda, "Wide-speed operation of interior permanent magnet synchronous motors with high-performance current regulator," IEEE Trans. Industry Applications, vol. 30, pp. 920 926, Jul./Aug. 1994.

[2] J.-K. Seok, J.-S. Kim, and S.-K. Sul, "Overmodulation strategy for highperformance torque control," IEEE Trans. Power Electronics, vol. 13, pp. 786-792, Jul. 1998.

[3] T. Gemaßmer, J. Richter, M. Schnarrenberger and M. Braun, "Dynamic overmodulation for highly dynamic current control of IPMSM with saturation characteristics," Int. Symposium on Power Electronics, Electric Drives, Automation and Motion, Ischia, Italy, 2014.

[4] Y.-C. Kwon, S. Kim, and S.-K. Sul, "Voltage feedback current control scheme for improved transient performance of permanent magnet synchronous machine drives," IEEE Trans. Industrial Electronics, vol. 59, pp. 3373-3380, Sep. 2012.

[5] S. Bolognani, L. Tubiana, and M. Zigliotto, "High dynamic PMSM current control by optimal saturation management of current regulators," $29^{\text {th }}$ Annual Conference of the IEEE Industrial Electronics Society, Roanike, USA, 2003.

[6] S. Bologna, M. Tomasini, L. Tubiana, and M. Zigliotto "Design and implementation of a minimum-time torque control for IPM motor drives," 40 $0^{\text {th }}$ IAS Annual Meeting. Conference Record of the 2005 Industry Applications Conference, Hong Kong, 2005.

[7] S. Lerdudomsak, S. Doki, and S. Okuma, "Voltage limiter calculation method for fast torque response of IPMSM in overmodulation range," $35^{\text {th }}$ Annual Conference of the IEEE Industrial Electronics Society, Porto, Portugal, 2009.

[8] H. Ghasemi, and S. Vaez-Zadeh, "A very fast direct torque control for permanent magnet synchronous motor start up," Canadian Conference on Electrical and Computer Engineering, Niagara Falls, Canada, 2004.

[9] J. S. Lee, R. D. Lorenz, and M. A. Valenzuela, "Time-optimal and lossminimizing deadbeat-direct torque and flux control for interior permanent-magnet synchronous machines," IEEE Trans. Industry Applications, vol. 50, pp. 1880-1890, May/Jun. 2014.

[10] J. Richter, T. Gemaßmer, and M. Doppelbauer, "Predictive current control of saturated cross-coupled permanent magnet synchronous machines," Int. Symposium on Power Electronics, Electric Drives, Automation and Motion, Ischia, Italy, 2014.

[11] J. Richter, A. Dollinger, and M. Doppelbauer, "Iron loss and parameter measurement of permanent magnet synchronous machines," $X X I^{s t}$ International Conference on Electrical Machines, Berlin, Germany, 2014.

[12] J. S. Lee, C. H. Choi, J. K. Seok, and R. D. Lorenz, "Deadbeat-direct torque and flux control of interior permanent magnet synchronous machines with discrete time stator current and stator flux linkage observer," IEEE Trans. Industry Applications, vol. 47, pp. 1749-1758, Jul./Aug. 2011. 